\title{
High Frequency of Fractures in an Early Stage of MS
}

\author{
Olga Krökki ${ }^{1,2}$, Risto Bloigu ${ }^{3}$ and Anne M Remes ${ }^{4,5^{*}}$ \\ ${ }^{1}$ Institute of Clinical Medicine, Neurology, University of Oulu, Oulu, Finland \\ ${ }^{2}$ Department of Neurology, Oulu University Hospital, Oulu, Finland \\ ${ }^{3}$ Medical Informatics Group, University of Oulu, Oulu, Finland \\ ${ }^{4}$ Institute of Clinical Medicine, Neurology, University of Eastern Finland, Kuopio, Finland \\ ${ }^{5}$ Department of Neurology, Kuopio University Hospital, Kuopio, Finland
}

${ }^{*}$ Corresponding author: Anne M Remes, Department of Neurology, Institute of Clinical Medicine, University of Eastern Finland, P.O. Box 1627, 70211 Kuopio, Finland, Tel: +358 447174655 ; Fax +358 17 172305; E-mail: anne.remes@uef.fi

Received date: Apr 11, 2014, Accepted date: June 16, 2014, Published date: June 20, 2014

Copyright: (C) 2014 Krokki O, et al. This is an open-access article distributed under the terms of the Creative Commons Attribution License, which permits unrestricted use, distribution, and reproduction in any medium, provided the original author and source are credited.

\begin{abstract}
Objective: Low-energy (fragility) fractures of the distal forearm, femur, and vertebrae are usually associated with osteoporosis. There is an increased risk of fractures and low bone mineral density in patients with MS, even during the early stages of the disease. We evaluated the prevalence and features of low-energy fractures in a populationbased MS cohort residing in a region of Finland near the Arctic.

Methods: The prevalence of fractures was determined in 491 patients who had been newly diagnosed with clinically definite MS. The fracture type, as well as the association between the duration of MS and the expanded disability status scale (EDSS) score, with a fracture event was determined.

Results: The prevalence of fragility fractures was $7.1 \%$. The most frequent fracture types were vertebral $(19.2 \%)$, distal forearm $(19.2 \%)$, and ankle $(25 \%)$, and the majority were low-energy fractures. The majority of fractures occurred within five years of MS diagnosis verification in non-disabled to moderately disabled patients (EDSS score $0-4)$. The mean MS patient age at which low-energy fracture events occurred was 42.5 years $(95 \% \mathrm{Cl}, 38.3-46.6$ years). Osteoporosis had only been clinically investigated in $26 \%$ of patients with fragility fractures, $89 \%$ of whom returned a positive finding.
\end{abstract}

Conclusions: The prevalence of low-energy vertebrae fractures was notably high in the present cohort; however, the presence of osteoporosis was poorly evaluated in clinical practice for this cohort.

Keywords: Fractures; Low-energy fracture; Bone density; Osteoporosis; Methylprednisolone; MS.

\section{Introduction}

In the developed world, $30 \%$ of women and $20 \%$ of men over the age of 50 years will sustain an osteoporosis-related fracture [1]. Lowtrauma or fragility fractures occur most commonly in the spine, proximal femur, or distal forearm, although they can also occur in the arm, pelvis, ribs, and other bones [2]. A history of a fragility fracture or fractures is predictive of future fractures [3]. Fragility fractures are associated with significant pain, suffering, disability and considerable costs to society [4].

Osteoporosis is defined as a generalised skeletal decrease in bone mineral density (BMD) and is an independent predictor of fragility fractures [4]. In patients with MS, reduced BMD [5], as well as an increased risk of falling [6], may increase the risk of fragility fractures, even in patients younger than 50 years old. Furthermore, the low vitamin D levels associated with MS may contribute to bone fragility; however, the evidence supporting this is inconclusive $[5,7,8]$. Population-based cohort studies from three European countries have revealed a higher risk of proximal femur fractures in patients with MS than in the general population. The overall risk of fractures is almost equivalent between MS and control subjects; however, the risk of clinical osteoporotic fractures tends to increase with increasing levels of disability [9-11]. Furthermore, a study from Norway shows that a high proportion of MS patients have osteoporosis or osteopenia when the disease is diagnosed, and this is even true of patients with clinically isolated syndrome [12]. The primary aim of this study was to evaluate the prevalence of low-energy fractures in an MS cohort residing in a region of Finland near to the Arctic. A secondary aim was to evaluate the expanded disability status scale (EDSS) level [13] and duration of MS at the time of a fragility fracture event.

\section{Methods}

\section{Study population}

The study was performed in the Outpatient Department of Neurology at Oulu University Hospital (OUH) with the approval of the Northern Ostrobothnia Ethics Committee and was designed according to the principles of the Declaration of Helsinki. Northern Ostrobothnia is a high-risk area for MS; it is situated in northern Finland, near to the Arctic Circle. The source population on the 31 December 2010 was 398,335 , amounting to $7.4 \%$ of the total population of Finland. The female/male ratio was 0.99 . Study data was 
obtained from hospital records, which contain broad information about patients with MS follow-up in OUH. Patients were included if they had been definitively diagnosed with MS between 1 January 1990 and 31 December 2010. All cases were identified from the hospital records by reference to code 3400A (MS) in ICD-9 (1 January 1992 to 31 December 1995) and code G35 (MS) in ICD-10 (1 January 1996 to 31 December 2010).

\section{Fractures}

All fracture types were identified from patients' hospital records. Patients who had sustained fractures before the age of 15 years or a cranial fracture at any age were excluded from the study. All fracture events were classified as high- or low-energy (fragility) fractures using the definitions of Flinkkila et al. [14] and Morrison et al. [15]. A highenergy injury was defined as that occurring from a sports activity, a fall from a height of more than $1 \mathrm{~m}$, a bicycle accident, a motor vehicle collision, or a major intrinsic event (e.g., a stroke, syncope, or epileptic seizure). Fractures that were not associated with these high-energy events were defined as low-energy fractures. A low-trauma fall was defined as a fall from standing height such as slipping, tripping, or stumbling on a flat surface or stairs. Non-fall fractures were also identified. A diagnosis of osteoporosis was confirmed from the hospital records and was based on the results of dual-energy X-ray absorptiometry (DXA) or an osteoporotic bone structure on X-rays. A subset of patients received a BMD examination for osteoporosis as part of their clinical treatment and investigations. A clinical osteoporotic fracture has been defined as a fracture of the radius, ulna, humerus, rib, femur, hip, pelvis, or vertebrae [16]. In the present study, clinical osteoporotic fractures were limited to those occurring in these anatomical locations for the purposes of comparison. None of the present cohort had rib or pelvic fractures. Fragility fractures of fingers, toes and ankle were excluded from calculations of clinical osteoporotic fractures.

\section{Statistical analysis}

All statistical analyses were performed using SPSS for Windows, (IBM Corp. Released 2011. IBM SPSS Statistics for Windows, version 20.0. Armonk, NY: IBM Corp). A P value of less than 0.05 was considered to be statistically significant. The baseline characteristics of MS patients with osteoporosis and fractures were compared using the Chi-square test. Scatter plot was used to analyse the association of disability and age at the time of a fracture event with the distribution of fractures according to fracture nature (low- vs. high-energy). To assess the association of steroid administration with the likelihood of a fracture, three subgroups were defined based on the administration profile of short courses of steroids during the follow-up: group 1, patients who did not receive steroids; group 2, patients who received fewer than five or five short courses of methylprednisolone; and group 3 , patients who received more than five short courses of methylprednisolone. Kaplan-Meier analysis was used to estimate the proportion of MS patients who sustained fractures during the early stage of MS and was performed because of differences in the follow-up duration after MS diagnosis.

\section{Results}

The study cohort consisted of 491 incident patients (69.2\% females) who were definitively diagnosed with MS during 1990-2010. Relapsing remitting or secondary progressive MS was diagnosed in $93.3 \%$ of cases. Primary progressive MS was diagnosed in $6.7 \%$ of cases. The mean age at the onset of MS was 32.0 years (range, 11-65 years). The mean age at the diagnosis of MS was 36.2 years (range, 12-70 years). There were no differences between genders in terms of their age at MS onset or diagnosis in the cohort in total.

We identified 39 MS patients (8.0\%) with at least one fracture event and a total of $52(10.6 \%)$ fracture events in the cohort. The first fracture event occurred within one year after MS diagnosis in 53.8\% $(n=21)$ of the 39 MS patients identified as having experienced at least one fracture event. Fourteen of those fractures occurred before the MS disease has been diagnosed. The percentage of patients experiencing a first fracture event cumulatively increased to $69.2 \%(n=27)$ within five years after the verified diagnosis of MS. According to a Kaplan-Meier analysis of the entire MS cohort, the probability of sustaining fractures within five, 10 , and 20 years after MS diagnosis were $5.7 \%, 6.9 \%$ and $14.2 \%$, respectively (Figure 1).

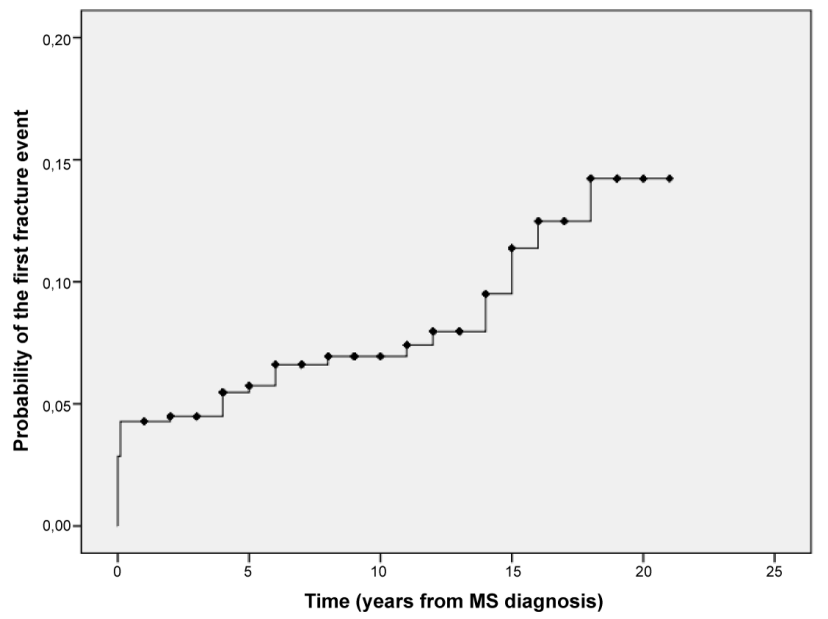

Figure1: The probability of sustaining the first fracture event after MS diagnosis in patients with MS.

The majority of patients $(n=28)$ with fractures experienced a single fracture event. However, nine patients sustained two fracture events, and two patients sustained three fracture events. The most frequent fracture sites were a single vertebra, distal forearm, ankle, and proximal femur (Table 1). 


\begin{tabular}{|c|c|c|c|c|c|}
\hline Type of fracture* & $\begin{array}{l}\text { Total fracture patients } \\
\qquad \mathbf{N}(\%)\end{array}$ & $\begin{array}{l}\text { Total fracture events } \\
\qquad \mathbf{N}(\%)\end{array}$ & $\begin{array}{l}\text { High-energy fracture } \\
\text { events } \\
\qquad \mathrm{N}(\%)\end{array}$ & $\begin{array}{l}\text { Low-energy fracture } \\
\text { events } \\
\qquad N(\%)\end{array}$ & $\begin{array}{l}\text { Fracture events from } \\
\text { the cohort \% }(\mathrm{N}=491)\end{array}$ \\
\hline Femur & $3(7.7)$ & $5(9.6)$ & $0(0)$ & $5(100)$ & 1.02 \\
\hline Humerus & $4(10.3)$ & $4(7.7)$ & $3(75)$ & $1(25)$ & 0.81 \\
\hline Vertebra & $9(23.0)$ & $10(19.2)$ & $4(40)$ & $6(60)$ & 2.04 \\
\hline Distal forearm & $8(20.5)$ & $10(19.2)$ & $4(40)$ & $6(60)$ & 2.04 \\
\hline Ankle & $8(20.5)$ & $13(25)$ & $3(23.1)$ & $10(76.9)$ & 2.65 \\
\hline Shin bone & $3(7.7)$ & $6(11.5)$ & $1(16.7)$ & $5(83.3)$ & 1.22 \\
\hline Finger, toe & $4(10.3)$ & $4(7.7)$ & $2(50)$ & $2(50)$ & 0.81 \\
\hline Total & $39(100)$ & $52(100)$ & $17(32.7)$ & $35(67.3)$ & 10.6 \\
\hline
\end{tabular}

Table 1: Characteristics of fractures. ${ }^{*}$ Cranial fractures and fracture age under 15 years are excluded.

Thirty-five fracture events (67.3\%) satisfied the criteria of a lowenergy fracture, and the overall frequency of low-energy fractures was $7.1 \%$. The majority of fragility fractures occurred in MS patients who were not disabled or mildly/moderately disabled (EDSS score $0-4$ ) (Figure 2).

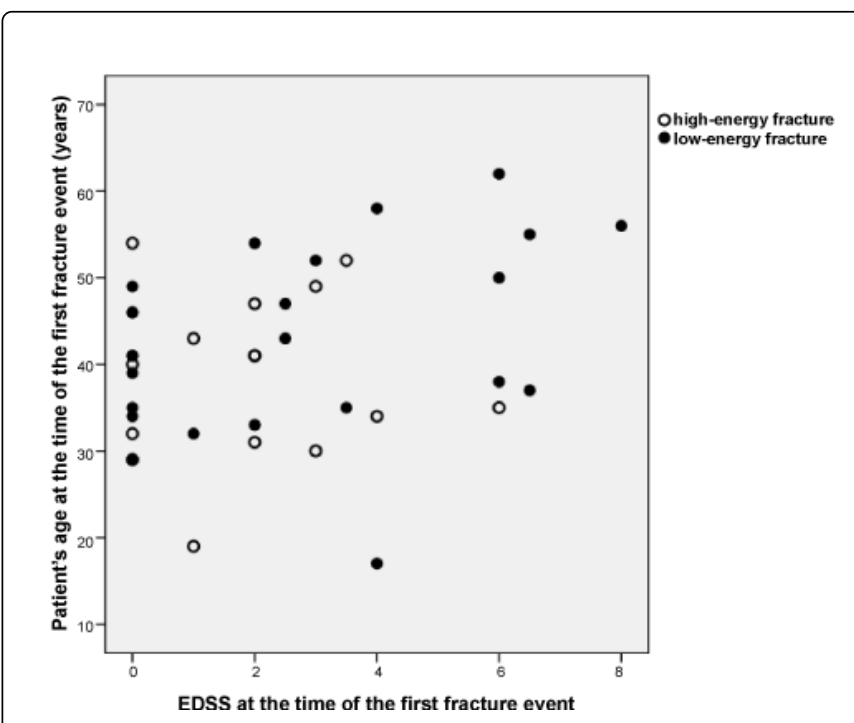

Figure2: The age and EDSS score of MS patients with low-energy and high-energy fractures.

There was no correlation between the location of the fracture and MS severity (Figure 3). Using the same criteria for osteoporotic fractures as previous registry studies, the prevalence of osteoporotic fractures was $5.9 \%(29 / 491)$. Of these fractures, 3.6\% $(n=18)$ were characterized as low-energy fractures. All of the femur fractures, $60 \%$ of the vertebral fractures, $77 \%$ of the ankle fractures, and $60 \%$ of the distal forearm fractures were classified as low-energy fractures.

Considering all fracture events, the mean age of patients when fractures occurred was 41 years (range, 17-62 years). The youngest patient had sustained a vertebral fracture and was diagnosed with osteoporosis at the age of 17 years; the patient had been diagnosed with MS 1 year before the fracture. The mean patient age at which high-energy and low-energy fracture events occurred was 37.1 years
(95\% CI, 31.3-43.0 years) and 42.5 years (95\% CI, 38.3-46.6 years), respectively. There was no significant difference between the age of patients who sustained fragility fractures and the age of patients who sustained high-energy fractures. Furthermore, when patients were grouped according to their gender or MS disease course, there were no significant intergroup differences in patient age at the time of their fracture. When patients were grouped according to their short courses of methylprednisolone administration profiles, there were no significant intergroup differences in the risk of fragility fractures.

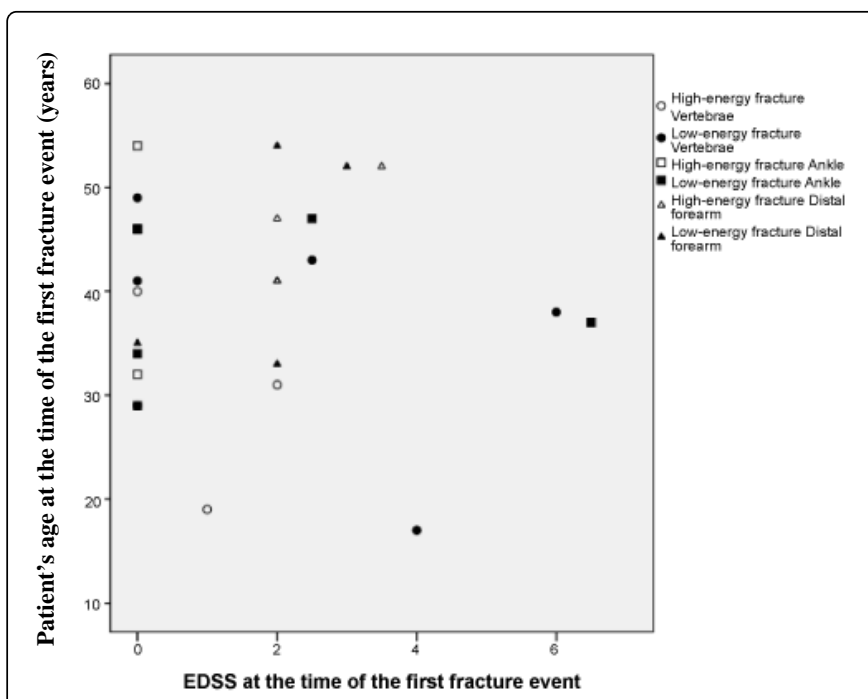

Figure 3: The age and EDSS score of MS patients with low-energy and high-energy fractures by their locations (vertebrae, distal forearm and ankle).

Patients who sustained low-energy fractures were not routinely investigated for osteoporosis. A DXA scan was only performed in $26 \%$ $(9 / 35)$ of fragility fracture cases, and the vast majority $(89 \%, n=8)$ of these patients were suffering from osteoporosis. An osteoporotic bone structure was specified in the $\mathrm{X}$-ray findings of one case. The majority of cases with fractures $(n=40 / 52)$ did not undergo any specific examination for osteoporosis. However, there was no mention of an osteoporotic bone structure in the X-ray findings of these cases. 


\section{Discussion}

The prevalence of fractures in this population-based Finnish Northern Ostrobothnia MS cohort was the same as that in the Danish registry study. Notably, more than two-thirds of all fracture events were fragility fractures in relatively young patients. The previously reported data on fracture risk in MS patients were obtained from three large databases: a UK general practice research database, the Danish National Health Registers, and the PHARMO Record Linkage System, which is linked to the hospitalization registry of the Netherlands $[9,16,17]$. In these studies, the prevalence of all fractures in patients with MS varied between $2 \%$ and $10 \%$. In these studies, osteoporotic fractures were defined based on anatomical location; however, patients were not routinely evaluated for osteoporosis using DXA. A notably increased risk of clinical osteoporotic proximal femoral fractures, and a slightly increased risk of pelvic, vertebral, and humeral fractures in MS patients compared to healthy controls has been reported $[16,18]$. In the present study, there was a comparatively high frequency of vertebral fractures in MS patients ( $2 \%$ in the present study vs. $0.3 \%$ in the Danish study). Vertebral fragility fractures are typically associated with older osteoporotic patients [19].Vertebral fragility fractures are a very strong risk factor for subsequent hip and vertebral fracture $[4,20]$. Only one population-based study on determinants of thoracic vertebral fractures in 57,480 Finnish population has been performed in the 1990s [21]. The prevalence of such fractures increased with age: after 40 years of age in the men and after 55 years of age in the women. In the patients aged 35-44 the prevalence of thoracic vertebral fractures was lower than in the present study. However, lumbar spine fractures were also included in our study. The previous epidemiological study did not focus on fracture nature as high- or lowenergy fractures. Furthermore, the epidemiology of vertebral fractures may have been changed over the past 20 years in general population in Finland.

According to Kaplan-Meier curves, MS patients seem to be at a higher risk of fractures at the first years from verified MS diagnosis and also 10 years after MS diagnosis probability of the first fracture event starts to grow up.

We also found that fractures occurred more commonly at a young age in MS patients that were not disabled or mildly/moderately disabled and only occasionally occurred in the advanced phase of the disease. Although the size of our cohort was limited, the study data included information on the duration, course, and level of disability associated with MS disease during the 21-year follow-up period and information on the time interval between fracture event and verified MS diagnosis. This enabled an evaluation of associations between patient age and disability level at the time of a fracture event as well as the nature of each fracture event (low- vs. high-energy). Reduced physical activity owing to high disability rates, fatigue, chronic inflammatory processes, vitamin D deficiency, and glucocorticoid use may increase the risk of osteoporosis and fractures in patients with MS [7]. Poor mobility and a high EDSS score level do not explain the high frequency of fragility fractures in the present study. Low BMD is prevalent in newly diagnosed MS patients with no or minor physical disability [12]. Patients from Finland already have low vitamin D levels owing to a deficit of solar radiation and a low vitamin D intake [22,23]. Mean vitamin D levels in the general Danish population have been reported to be sufficient [24].The low level of vitamin D in Finnish population could increase the risk of osteoporosis and may partly explain the higher number of vertebrae fractures in our MS cohort compared to Danish database.
It was not possible to evaluate the prevalence of osteoporosis in our cohort because this condition was not routinely tested for. No data are available on the prevalence of osteoporosis in Finnish MS patients. In the United States, the prevalence of osteoporosis in MS patients has been estimated to be $15.4 \%$ [25]. It has been recommended that MS patients with high levels of disability (EDSS score $>6$ ), especially postmenopausal women, are screened for osteoporosis using DXA [7]. Osteoporosis screening for MS patients has also been recommended within 2 years of MS diagnosis [26]. A timely assessment of fracture risk and subsequent treatment of osteoporosis may prevent fractures and ameliorate the quality of life of MS patients.

Patients with MS are at an increased risk of fractures. Osteoporosis may be a major factor predisposing MS patients in the early stage of the disease to low-energy fractures. MS patients should be more systematically investigated for osteoporosis. Effects of osteoporosis on MS patients warrant further investigation.

\section{Funding}

This work was supported financially by clinical EVO grants from Oulu University Hospital.

\section{References}

1. Baim S, Leslie WD (2012) Assessment of fracture risk. Curr Osteoporos Rep 10: 28-41

2. National Clinical Guideline Centre (UK) ( 2012) Osteoporosis: Fragility Fracture Risk: Osteoporosis: Assessing the Risk of Fragility Fracture. NICE Clinical Guidelines, No. 146. Royal College of Physicians (UK), London.

3. Kanis JA, Johnell O, De Laet C, Johansson H, Oden A et al. (2004) A meta-analysis of previous fracture and subsequent fracture risk. Bone 35: 375-382.

4. Hernlund E, Svedbom A, Ivergard M, Compston J, Cooper C et al. (2013) Osteoporosis in the European Union: medical management, epidemiology and economic burden. Arch.Osteoporos 8: 136.

5. Ozgocmen S, Bulut S, Ilhan N, Gulkesen A, Ardicoglu O et al. (2005) Vitamin D deficiency and reduced bone mineral density in multiple sclerosis: effect of ambulatory status and functional capacity. J Bone Miner Metab 23: 309-313.

6. Tremlett H, Lucas R (2012) The risks for falls and fractures in multiple sclerosis. Neurology 78: 1902-1903.

7. Hearn AP, Silber E (2010) Osteoporosis in multiple sclerosis. Mult Scler 16: 1031-1043.

8. Dobson R, Ramagopalan S, Giovannoni G (2012) Bone health and multiple sclerosis. Mult Scler 18: 1522-1528.

9. Bazelier MT, van Staa T, Uitdehaag BM, Cooper C, Leufkens HG et al (2011) The risk of fracture in patients with multiple sclerosis: the UK general practice research database. J Bone Miner Res 26: 2271-2279.

10. Bazelier MT, de Vries F, Bentzen J, Vestergaard P, Leufkens HG et al. (2012) Incidence of fractures in patients with multiple sclerosis: the Danish National Health Registers. Mult Scler 18: 622-627.

11. Bazelier MT, van Staa TP, Uitdehaag BM, Cooper C, Leufkens HG et al. (2012) A simple score for estimating the long-term risk of fracture in patients with multiple sclerosis. Neurology 79: 922-928.

12. Moen SM, Celius EG, Sandvik L, Nordsletten L, Eriksen EF et al. (2011) Low bone mass in newly diagnosed multiple sclerosis and clinically isolated syndrome. Neurology 77: 151-157.

13. Kurtzke JF (1983) Rating neurologic impairment in multiple sclerosis: an expanded disability status scale (EDSS). Neurology 33: 1444-1452.

14. Flinkkila T, Sirnio K, Hippi M, Hartonen S, Ruuhela R et al. (2011) Epidemiology and seasonal variation of distal radius fractures in Oulu, Finland. Osteoporos Int 22: 2307-2312. 
Citation: Krökki O, Bloigu R, Remes AM (2014) High Frequency of Fractures in an Early Stage of MS . J Mult Scler 1: 109. doi:10.4172/jmso. 1000109

Page 5 of 5

15. Morrison A, Fan T, Sen SS, Weisenfluh L (2013) Epidemiology of falls and osteoporotic fractures: a systematic review. Clinicoecon Outcomes Res 5: 9-18.

16. Bazelier MT, Bentzen J, Vestergaard P, Stenager E, Leufkens HG et al. (2012) The risk of fracture in incident multiple sclerosis patients: the Danish National Health Registers. Mult Scler 18: 1609-1616.

17. Bazelier MT, van Staa TP, Uitdehaag BM, Cooper C, Leufkens HG et al. (2012) Risk of fractures in patients with multiple sclerosis: a populationbased cohort study. Neurology 78: 1967-1973.

18. Ramagopalan SV, Seminog O, Goldacre R, Goldacre MJ (2012) Risk of fractures in patients with multiple sclerosis: record-linkage study. BMC Neurol 12: 135-2377-12-135.

19. Lespessailles E, Cotte FE, Roux C, Fardellone P, Mercier F et al. (2009) Prevalence and features of osteoporosis in the French general population: the Instant study. Joint Bone Spine 76: 394-400.

20. Melton LJ,3rd, Atkinson EJ, Cooper C, O'Fallon WM, Riggs BL (1999) Vertebral fractures predict subsequent fractures. Osteoporos Int 10: 214-221.

21. Santavirta S, Konttinen YT, Heliovaara M, Knekt P, Luthje P et al. (1992) Determinants of osteoporotic thoracic vertebral fracture. Screening of 57,000 Finnish women and men. Acta Orthop Scand 63: 198-202.
22. Huotari A, Herzig KH (2008) Vitamin D and living in northern latitudes--an endemic risk area for vitamin $\mathrm{D}$ deficiency. Int $\mathrm{J}$ Circumpolar Health 67: 164-178.

23. Kauppi M, Impivaara O, Maki J, Heliovaara M, Marniemi J et al. (2009) Vitamin D status and common risk factors for bone fragility as determinants of quantitative ultrasound variables in a nationally representative population sample. Bone 45: 119-124.

24. Johansen JV, Manniche C, Kjaer P (2013) Vitamin D levels appear to be normal in Danish patients attending secondary care for low back pain and a weak positive correlation between serum level Vitamin D and Modic changes was demonstrated: a cross-sectional cohort study of consecutive patients with non-specific low back pain. BMC Musculoskelet Disord 14: 78.

25. Marrie RA, Cutter G, Tyry T, Vollmer T (2009) A cross-sectional study of bone health in multiple sclerosis. Neurology 73: 1394-1398.

26. Kampman MT, Eriksen EF, Holmoy T (2011) Multiple sclerosis, a cause of secondary osteoporosis? What is the evidence and what are the clinical implications? Acta Neurol Scand Suppl $191: 44-49$. 